\title{
Éditorial - Une nouvelle équipe éditoriale pour Économie rurale
}

Pierre labarthe et Aude Ridier

\section{Q OpenEdition}

1 Journals

\section{Édition électronique}

URL : http://journals.openedition.org/economierurale/3885

DOI : 10.4000/economierurale.3885

ISSN : 2105-2581

Éditeur

Société Française d'Économie Rurale (SFER)

\section{Édition imprimée}

Date de publication : 15 mars 2013

Pagination : 4-5

ISSN : 0013-0559

\section{Référence électronique}

Pierre labarthe et Aude Ridier, «Éditorial - Une nouvelle équipe éditoriale pour Économie rurale », Économie rurale [En ligne], 334 | mars-avril 2013, mis en ligne le 15 mars 2015, consulté le 24 septembre 2020. URL : http://journals.openedition.org/economierurale/3885 ; DOI : https://doi.org/ 10.4000/economierurale.3885 


\section{ÉDITORIAL}

\section{Une nouvelle équipe éditoriale pour Économie rurale}

C omme annoncé dans l'éditorial du numéro 333, une nouvelle équipe est désormais en charge de la gestion éditoriale et de l'animation scientifique de la revue. Nous tenons à remercier Bernard Roux et les membres de l'équipe précédente pour tout le travail accompli au sein du Comité de rédaction et du secrétariat éditorial. La nouvelle équipe affiche, dès sa composition, la volonté réaffirmée de la revue d'embrasser non seulement la diversité des approches et des champs thématiques de «l'économie rurale » (l'agriculture, l'alimentation, l'organisation des filières, l'espace rural, l'environnement...) mais aussi celle des disciplines des sciences humaines et sociales (économie, sociologie, géographie, sciences politiques). Le premier objectif du nouveau Comité sera d'améliorer l'attractivité de la revue pour des contributions de langue française, en veillant à la qualité scientifique des articles publiés ainsi qu'à la qualité de la relation avec les auteurs (transparence, réactivité, dialogue). Pour cela, la dimension collective du fonctionnement éditorial a été renforcée grâce à la participation accrue de l'ensemble des membres du Comité aux décisions éditoriales. La revue, qui occupe une part importante au sein des activités de la Société française d'économie rurale (SFER), rejoint les objectifs de partage et de diffusion de connaissances de l'association. Le nouveau Comité éditorial sera donc particulièrement attentif à la lisibilité des articles pour différents publics (chercheurs et enseignants de différentes disciplines, décideurs publics, acteurs du développement agricole et rural...) et à la qualité du contenu empirique des contributions.

Le processus de production de la revue a aussi été transformé pour tendre à plus d'autonomie financière. La fabrication d'Économie rurale, sous format papier et électronique (consultable sur revues.org et sur cairn.info), est désormais déléguée à l'éditeur CAIRN. Cette nouvelle collaboration nous permet également de bénéficier d'un appui professionnel dans la relecture des articles, et de compenser ainsi le départ progressif de Nicole Bauchet, que nous tenons à remercier chaleureusement pour son implication dans la vie de la revue et pour tout le travail qu'elle a accompli bénévolement durant de nombreuses années. Dans ce nouveau fonctionnement, la direction scientifique ainsi que la gestion et l'animation éditoriale restent sous l'entière responsabilité du Comité éditorial. Elles continueront à s'appuyer sur le professionnalisme et la qualité du travail de secrétariat éditorial réalisé par Sandrine Corcuff.

La construction de ce numéro 334 s'inscrit dans une phase de transition entre les équipes éditoriales : il rassemble des contributions sélectionnées et suivies par l'ancien Comité de rédaction, que nous remercions de ce travail. Les articles que nous avons choisi d'y regrouper témoignent de la diversité des thèmes, des contextes et des problématiques socio-économiques, mais aussi de la diversité des approches disciplinaires que nous voulons placer au cœur de la ligne éditoriale de la revue. Différentes disciplines sont ainsi représentées : l'économie, avec l'article de 
Blancard, Boussemart, Flahaut et Lefer qui propose une nouvelle approche pour mesurer les performances productives des exploitations agricoles; les sciences de gestion avec l'article de Simon, qui analyse les intentions et les perceptions d'étudiants vis-à-vis de l'installation comme agriculteur ; la géographie avec l'article de Quintal et Trudelle, qui porte sur le rôle des associations locales dans l'action collective pour la gouvernance territoriale et la gestion de ressources au sein de communautés oasiennes au Maroc ; ainsi que celui de Bonnefond et Fournier, sur le rôle de l'action publique dans la maîtrise foncière des espaces ruraux, à partir du cas d'un contrat de rivière en France ; et la sociologie, avec l'article de Moumouni centré sur la perception de différents acteurs, publics ou privés, vis-à-vis de la participation financière des producteurs aux services de conseil agricole dans les contextes d'Afrique subsaharienne (Bénin).

Ce numéro présente également des contributions dans les rubriques Faits et chiffres et Débat, dont les contenus ont évolué avec les objectifs d'échange de connaissances de la SFER. L'article de Fèvre et Pouch présente un état des lieux en Faits et chiffres sur un enjeu important : I'affirmation des multinationales de l'agroalimentaire des pays émergents sur les marchés mondiaux, illustrée à partir du cas des firmes brésiliennes dans la filière viande. La rubrique Débat s'appuie désormais sur les Séminaires de politique agricole (SPA) de la SFER, animés par Aurélie Trouvé et Frédéric Courleux. Elle propose, dans ce numéro, le croisement de deux points de vue librement exprimés sur les projets de réforme de la Politique agricole commune après 2013 : celui de Tomás García Azcárate, fonctionnaire à la Commission européenne et membre de l'Institut d'études européennes, et celui de Régis Hochart, représentant de la Confédération paysanne au Conseil économique, social et environnemental. Enfin, les Notes de lecture resteront un rendez-vous régulier pour nos lecteurs.

Nous voulons remercier les membres de la nouvelle équipe qui ont déjà manifesté une forte mobilisation pour inscrire durablement la revue dans le paysage des revues scientifiques francophones, ainsi que les auteurs et relecteurs qui permettront à Économie rurale de proposer, dans une perspective pluridisciplinaire, un regard original sur les nouvelles transformations des agricultures, des territoires et de l'alimentation.

Pierre Labarthe et Aude Ridier Directeurs du Comité éditorial

Retrouvez Économie rurale en ligne sur www.cairn.info portail de revues en sciences humaines et sociales, en texte intégral, à partir du premier numéro de 2009 jusqu'au dernier numéro paru et sur revue.org de 2005 à 2008. 